\title{
Incidence in dialysis and chronic kidney disease prevalence in the Portuguese population
}

\author{
M. Martins Prata \\ Professor of Nephrology
}

Portugal has the highest incidence of dialysis in Europe and one of the highest in the world. The cause or causes are not completely understood. ${ }^{1}$

However, it is easy to understand that a high prevalence of risk factors for chronic kidney disease (CKD) and its rate of progression could explain a high prevalence of CKD and, as a possible consequence, a higher rate of dialysis incidence. So, it is quite understandable how important it would be to demonstrate the existence of a high prevalence of CKD in the Portuguese population. Indeed, it is a key public health and epidemiological research issue. ${ }^{2}$

Unfortunately, until recently, we had only one study - PREVADIAB Study ${ }^{3}$ - and this showed a CKD prevalence in the Portuguese population of $6.1 \%$, similar to that observed in other countries where the incidence of dialysis is much lower.

More recently, in October 2020, a second study was published, in Nephron: "RENA Study: cross-sectional study to evaluate CKD prevalence in Portugal". ${ }^{4}$ It is hard to understand how the referees of this journal accepted a paper with such an inappropriate title. The study was based on a sample of the population that is absolutely non-representative of the Portuguese population.

The main goal of the study was clearly defined: "to overcome the limitations of the PREVADIAB study, the RENA study aimed to provide an estimate of the prevalence of CKD at a national level and to characterize CKD patients". Surprisingly, the study included randomly selected patients, aged 18 or more, which were present in the waiting rooms of Primary Care Health Units (PCHU), on pre-specified days. Therefore, the study did not include a random sample of the general population. The authors themselves considered that PCHU attendees were not representative of the Portuguese population (more women, older ages, multiple comorbidities, and possibly less healthy than the general population). To overcome this selection bias, data adjustments were made, but the authors agreed that they cannot measure the impact of the extrapolation for the overall population.

It was easily and comprehensively predictable, even before the beginning of the analysis of the data obtained, that the CKD prevalence in this sample would be, most probably, different and higher than that actually existing in the Portuguese population.

The study has other limitations: the population of large regions of the country was not included, such as the Northern Region that comprises more than one-third of the country's population.
The CKD1-5 prevalence estimated in this unhealthy Portuguese population was $20.7 \%$, a level in the higher range of others observed in several countries. However, this value includes a higher proportion of CKD stages 1 and 2, situations that are more frequent in elderly people and usually with a better prognosis.

Moreover, it is also important to know that no correlation was observed between the CKD prevalence estimated by this study and the incidence in dialysis in the same regions of the country where the study was carried out. ${ }^{5}$ In conclusion, the results of this study are not credible and, in any case, do not allow us to conclude that the high CKD prevalence is the main, or the unique factor, responsible for the high incidence of dialysis in Portugal. We must agree that a more reliable study is needed.

Although we believe that given such high levels of risk factors for CKD - diabetes, hypertension, obesity, high sodium diet, tobacco use, and physical inactivity - we certainly have a high CKD prevalence, we must produce strong evidence not only about the existence of a high prevalence of $\mathrm{CKD}^{3-5}$ in the Portuguese population but also that these values are the only factors responsible for the high incidence in dialysis or if others coexist and what their relevance is.

Furthermore, we need to keep in mind that, if, on the one hand, the existence of a high prevalence of CKD may be the cause or one of the causes of the high prevalence in dialysis in Portugal, this high value of prevalence of CKD is, with a great probability, a strongly negative indicator of our performance as doctors and as nephrologists.

A quality replacement therapy for kidney function, such as the one our patients enjoy, is a given. The objective has become to significantly reduce the number of patients who need to use this type of therapy every year.

\section{References}

1. M. Martins Prata. "Incidence in dialysis in Portugal and Spain" Edition of the author (request by email mateus.mprata2@gmail.com).

2. Stel VS, Brück K, Fraser S, Zoccali C, Massy ZA, Jager KJ. International differences in chronic kidney disease prevalence: a key public health and epidemiological research issue. Nephrol Dial Transpl 2017;32:129-135

3. Vinhas J, Gardete-Correia L, Boavida JM, Raposo JF, Mesquita A, Fona MC et al. Prevalence of chronic kidney disease and associated risk factors, and risk of end-stage renal disease: data from the PREVADIAB study. Nephron Clin Pract 2011:119(1):c35-c40

4. Vinhas J, Aires I, Batista C, Branco P, Brandão J, Nogueira R, Raposo JF, Rodrigues E. RENA study: Cross-sectional study to evaluate CKD prevalence in Portugal.Nephron 2020;144(10):479-487

5. De Almeida E, Raimundo M, Coelho A, Sá $H$. Incidence, prevalence and crude survival of patients starting dialysis in Portugal (2010-2016): analysis of the National Health System individual registry. Clin Kidney J 2020;14(3):869-875. 